\title{
The Place of Meditation in the Health Care System
}

\section{Daniel Neto*}

Serviço de Saúde da RegiãoAutónoma da Madeira, EPE, Portugal

\begin{abstract}
The conclusions of the work published in PLoSOne: "Unwanted effects: Is there a negative side of meditation? A multicenter survey" should open a debate about the place of different practices in the health care system and future research. There are plenty of works being done, a lot of them with biased designs, showing benefits. This work shows a different point, about a poorly researched area within meditation. All the interventions are subjected to side effects.
\end{abstract}

\section{Publication History:}

Received: May 30, 2018

Accepted: July 24, 2018

Published: July 26, 2018

Keywords:

Meditation, Mindfulness, Psychotherapy, spiritual practices
In recent years, meditation gained the hotspot of psychological recommendations, even making to the recommendations of the NICE Guidelines (in the version of mindfulness) [1]. Multiple benefits were described and studied, among them are reducing of stress, depression, anxiety and its effects on chronic illnesses [2].

"Unwanted effects: Is there a negative side of meditation? A multicenter survey" analysis the question of its side effects [3].

It's important to reflect on the origins of meditation and its adoption by the health care system and scientific community. Meditation was born in the spiritual practices and had a main goal: to raise human awareness through the training of the human mind. Some view this as old psychological tools, but it is far more then it. It is integrated in a complex system for human enlightenment.

It's important to reflect on the origins of meditation and its adoption by the health care system and scientific community. Meditation was born in the spiritual practices and had a main goal: to raise human awareness through the training of the human mind. Some view this as old psychological tools, but it is far more then it. It is integrated in a complex system for human enlightenment.

The conceptualization that meditation is an old psychotherapeutic tool is very disturbing, because meditation born outside of any psychological school. As such, our understating of meditation is bonded to our understanding of its origin and context, not by its function. To understand mindfulness, and meditation, we need to study within the spirituality community. And I dare even say, maintain it within the inner development (spiritual) dimension of humanity. Of course meditation could be studied, but its practice should be regarded as an inner development and not a symptomatic treatment.

The study presents this group of side effects from a checklist:

1. Greater emotional pain; More fear/anxiety/depression; Restlessness/anxiety when not practicing formal meditation; Greater self-criticism; Greater mental confusion;

2. Lack of life orientation; Less motivation in life; Feeling of needing something more; of lacking something; etc; Need for continuous meditation; Feeling that the time not spent meditating is wasted;

3. Increased criticism of others; Greater awareness of their negative traits; Feeling of being superior to them/better than them; Feelings of boredom caused by people; Feelings of lack of interest in others; Lack of interest in people's conversations; Feeling that only people who meditate are valuable;
4. Feeling of being alienated from society; Hypersensitivity/ rejection of urban life; Difficulty in feeling comfortable in the world. Lack of interest in your surroundings;

5. Boredom;

These side effects are very interesting. The group (1) reflects the absence of a therapeutic effect. If we understand that meditation is not a therapeutic tool, we understand that meditation should not make people feel better. Meditation is a confrontation with the inner self. And most of the times its not comfortable. The group (2) reflects people searching in meditation a solution for their life's. Mediation is a tool in a certain path, not a solution per se. The group (3) shows the wrong way to use meditation. It happens in people beginning their spiritual path and shows a superficial understanding of the process. We should remember that it also happens to a lot of people undergoing psychotherapy. The group (4) shows people using meditation that probably are already with some degree of disconnection with the world. This happens usually in people with depression, but also in people going throw the spiritual process, using it to distance themselves from the problems of daily life. The group (5) shows the people who probably are not ready for a meditation practice at the moment.

The increased side effects regarding higher frequency, higher duration of the session or in individual practice are clear associated to exposure. The bigger exposure to something not adequate or without the proper orientation has more frequent and more intense side effects.

From the spiritual practices we better understand the side effects [6]. The inner tackle of one's mind is a powerful but disturbing thing. Every negative aspect floats to the mind's eye first.

From the spiritual context, we understand the contraindications of meditation are not impeditive of its practice, but it should be with skilled teachers. Some individuals are not suited to meditation according to their own temperamental characteristics. Individuals

*Corresponding Author: Dr. Daniel Neto, Serviço de Saúde da RegiãoAutónoma da Madeira, EPE, Portugal; E-mail: danielcarvalhoneto@gmail.com

Citation: Neto D (2018) The Place of Meditation in the Health Care System. Int J Psychol Behav Anal 4: 147. doi: https://doi.org/10.15344/2455-3867/2018/147

Copyright: (C) 2018 Neto. This is an open-access article distributed under the terms of the Creative Commons Attribution License, which permits unrestricted use, distribution, and reproduction in any medium, provided the original author and source are credited. 
with severe psychotic illnesses should only be allowed to do such practices with senior and advanced teachers with strong sensibility to mental illness. Agitation during the practice that the individual is not capable of reducing by its own will is a momentary contraindication and the individual should not struggle to continue. If a person is not comfortable with the teacher, the practice or anything else during meditationshouldstopandseekotherprofessionalsorotheropinions [6].

Recognized practices that are associated with harmful effects are the kundalini meditations, breathing exercises, long fasting, sensorial and social deprivation and meditations with drugs. Within the spiritual community its recognized that every practice has its own place in the inner development and that they should not be used without proper understanding from the teacher and from the student. Quality teachers are sparse in every unregulated community [6].

The final risk is the risk of the professionals. Its common knowledge that a good tool is a tool developed and trained trough time. The integrative approach to mental health is very seductive, but the greatest side effect is superficiality of the training. Future trainees are directed at integration, because senior professionals understood that different schools are complementary to each other. But the trainee is far from the profound knowledge of the senior and easily jumps to simple conclusions that are very dangerous. We are in need of deep tools to understand psychological interventions true harm potential (not just worsening anxiety/depression scales or present subtle symptoms).

Meditation should be developed and trained, in the proper setting. Meditation is not a psychological tool.

\section{Competing Interests}

The authors declare that they have no competing interests.

\section{References}

1. National Collaborating Centre for Mental Health (2009) Depression in adults: recognition and management. Clinical guideline [CG90]. Leicester and London (UK): British Psychological Society and The Royal College of Psychiatrists.

2. Bohlmeijer E, Prenger R, Taal E, Cuijpers $P$ (2010) The effects of mindfulnessbased stress reduction therapy on mental health of adults with a chronic medical disease: A meta-analysis. J Psychosom Res 68: 539-544.

3. Cebolla A, Demarzo M, Martins P, Soler J, Garcia-Campayo J, et al. (2017) Unwanted effects: Is there a negative side of meditation? A multicenter survey. PLoS ONE 12: e0183137.

4. Walsh R, Rochs L (1979) Precipitation of acute psychotic episodes by intensive meditation in individuals with a history of schizophrenia. American Journal of Psychiatry 136: 1085-1086.

5. French AP, Schmid AC, Ingalls E (1975) Transcendental meditation, altered reality testing, and behavioral change: a case report. J NervMent Dis 161 55-58.

6. del Pe (2015) Hidden Dangers of Meditation and Yoga: How to Play with Your Sacred Fires Safely. Mdp Global Resources. 